\title{
Aurora-A overexpression is linked to development of aggressive teratomas derived from human iPS cells
}

\author{
SEIGA OHMINE ${ }^{1}$, JEFFREY L. SALISBURY ${ }^{3}$, JAMES INGLE ${ }^{2}$, GIUSEPPE PETTINATO ${ }^{4}$, CANDACE L. HADDOX ${ }^{2}$, \\ TUFIA HADDAD $^{2}$, EVANTHIA GALANIS $^{1,2}$, YASUHIRO IKEDA ${ }^{1 *}$ and ANTONINO B. D'ASSORO ${ }^{2,3 *}$ \\ Departments of ${ }^{1}$ Molecular Medicine, ${ }^{2}$ Medical Oncology and ${ }^{3}$ Biochemistry and Molecular Biology, \\ Mayo Clinic College of Medicine and Science, Rochester, MN 55905; ${ }^{4}$ Department of Surgery, \\ Beth Israel Deaconess Medical Center, Harvard Medical School, Boston, MA 02215, USA
}

Received May 31, 2017; Accepted December 7, 2017

DOI: $10.3892 /$ or.2018.6239

\begin{abstract}
The discovery of human induced pluripotent stem cells (hiPSCs) is a promising advancement in the field of regenerative and personalized medicine. Expression of SOX2, KLF4, OCT4 and MYC transcription factors induces the nuclear reprogramming of somatic cells into hiPSCs that share striking similarities with human embryonic stem cells (hESCs). However, several studies have demonstrated that hESCs and hiPSCs could lead to teratoma formation in vivo, thus limiting their current clinical applications. Aberrant cell cycle regulation of hESCs is linked to centrosome amplification, which may account, for their enhanced chromosomal instability (CIN), and thus increase their tumorigenicity. Significantly, the tumor suppressor p53 plays a key role as a 'guardian of reprogramming', safeguarding genomic integrity during hiPSC reprogramming. Nevertheless, the molecular mechanisms leading to development of CIN during reprogramming and increased tumorigenic potential of hiPSCs remains to be fully elucidated. In the present study, we analyzed CIN in hiPSCs derived from keratinocytes and established that chromosomal and mitotic aberrations were linked to centrosome amplification, Aurora-A overexpression, abrogation of p53-mediated G1/S cell cycle checkpoint and loss of Rb tumor-suppressor function. When hiPSCs were transplanted into the kidney capsules of immunocompromised mice, they developed high-grade teratomas characterized by the presence of cells that exhibited non-uniform shapes and sizes, high nuclear pleomorphism and centrosome amplification. Significantly, ex vivo cells derived from teratomas exhibited
\end{abstract}

Correspondence to: Dr Antonino B. D'Assoro, Department of Medical Oncology, Mayo Clinic College of Medicine and Science, 200 First Street SW, Rochester, MN 55905, USA

E-mail: dassoro.antonio@mayo.edu

*Contributed equally

Key words: hiPSCs, cell cycle checkpoint, Aurora-A, teratoma, metastasis high self-renewal capacity that was linked to Aurora-A kinase activity and gave rise to lung metastasis when injected into the tail vein of immunocompromised mice. Collectively, these findings demonstrated a high risk for malignancy of hiPSCs that exhibit Aurora-A overexpression, loss of Rb function, centrosome amplification and CIN. Based on these findings, we proposed that Aurora-A-targeted therapy could represent a promising prophylactic therapeutic strategy to decrease the likelihood of CIN and development of aggressive teratomas derived from hiPSCs.

\section{Introduction}

The discovery of human induced pluripotent stem cells (hiPSCs) has revolutionized the field of pluripotent stem cell research (1). Expression of OCT4 and SOX2, genes involved in early development, in concert with MYC and KLF4 oncogenes, can induce the transformation of adult somatic cells into hiPSCs that adopt morphological and functional characteristics of pluripotency indistinguishable from human embryonic stem cells (hESCs) $(2,3)$. The importance of obtaining hiPSCs that possess the same phenotype as hESCs, resides in the fact that such cells can be differentiated into tissues derived from the three germ layers when appropriate differentiation protocols are applied (4-6). Although hiPSCs represent an important source for therapeutic applications such as regenerative and personalized medicine (7-9), one of the major hindrances in translating the hiPSC technology into the clinic is the development of teratoma-like tumors that originate from the in vivo growth of hiPSCs (10). For this reason, before hiPSCs can be successfully used in innovative therapeutic designs, it is mandatory to understand their karyotypic stability. Notably, forced expression of OCT4, SOX2, MYC and KLF4 reprogramming factors has been revealed to predispose hiPSCs to chromosomal aberrations (11). Impairment of the cell cycle machinery has an important role in the propensity of hESCs and hiPSCs to acquire chromosomal defects. It has been demonstrated that aberrant cell cycle regulation of hESCs is linked to numerical centrosomal abnormalities during mitosis, which may account, for their enhanced chromosomal instability (CIN), and thus increase their tumorigenicity (12). Supporting these findings, the tumor suppressor p53 plays a key role as a 
'guardian of reprogramming', safeguarding genomic integrity during reprogramming at the cost of a reduced efficiency of the process (13). Notably, the mitotic kinase Aurora-A, that induces centrosome amplification and CIN in cancer (14), facilitated pluripotency through phosphorylation-mediated inhibition of p53-directed ectodermal and mesodermal gene expression (15). Phosphorylation of p53 not only impaired p53-induced hESC differentiation but also p53-mediated suppression of hiPSC reprogramming. Although these studies demonstrated a critical role for the Aurora-A/p53 axis in the regulation of self-renewal, chromosomal stability and somatic cell reprogramming, it remains to be explored whether concurrent aberrant Aurora-A activity and loss of p53 function increases the tumorigenicity of hiPSCs.

In the present study we analyzed the development of CIN during hiPSCs reprogramming and demonstrated that chromosomal and mitotic aberrations were linked to centrosome amplification, Aurora-A overexpression, abrogation of p53-mediated G1/S cell cycle checkpoint and loss of Rb function. Notably, hiPSCs with CIN-developed high-grade teratomas harboring centrosome abnormalities in immunocompromised mice and ex vivo teratoma cells exhibited high self-renewal capacity in vitro that was linked to Aurora-A kinase activity and development of lung metastasis. Collectively, these findings demonstrated a previously undisclosed linkage between Aurora-A overexpression, CIN and development of aggressive teratomas derived from hiPSCs.

\section{Materials and methods}

Generation of human iPS cells (hiPSCs). hiPSCs from skinderived keratinocytes (N1-hiPSCs) and blood-derived cells (DS1-hiPSCs) were established by transduction of 4 reprogramming lentiviral vectors as previously described (16). hiPSCs were maintained in Pluriton Reprogramming Medium (Stemgent, Cambridge, MA, USA) supplemented with 25\% (v/v) mTeSR ${ }^{\mathrm{TM}}-1$ maintenance media (Stemcell Technologies, Vancouver, BC, Canada) on BD Matrigel-coated cell culture plates (BD Biosciences, San Jose, CA, USA).

Spectral karyotyping (SKY) analysis. Hybridization, wash, and detection of the human SKYPaint ${ }^{\circledR}$ probe (Applied Spectral Imaging, Vista, CA, USA) were performed as recommended by the manufacturer. Image acquisition and spectral analysis of metaphase cells were achieved by using the SD200 SpectraCube ${ }^{\mathrm{TM}}$ Spectral Imaging system (Applied Spectral Imaging) mounted on a Zeiss Axioplan2 microscope (Carl Zeiss MicroImaging, Inc., Thornwood, NY, USA). Images were analyzed using HiSKY analysis software (Applied Spectral Imaging).

Immunoblotting, immunofluorescence and FACS assays. Immunoblotting, immunofluorescence and FACS assays were performed as previously described (14). Antibodies that were employed to perform these studies were the following: p53 (1:500; mouse monoclonal; cat. no. PIMA512557; Invitrogen/ Thermo Fisher Scientific, Waltham, MA, USA), p21 (1:500; mouse monoclonal; cat. no. AHZ0422; Thermo Fisher Scientific), centrin $20 \mathrm{H} 5$ (1:1,000; mouse monoclonal; Mayo Clinic, Rochester, MN, USA), pericentrin (1:500; rabbit polyclonal; cat. no. ab44481) and p-retinoblastoma (1:400; rabbit polyclonal; cat. no. ab47763; both from Abcam, Cambridge, MA, USA), $\gamma$-tubulin (1:4,000; mouse monoclonal; cat. no. MA1-850; Thermo Fisher Scientific), Aurora-A (1:500; mouse monoclonal; cat. no. ab13824; Abcam), p-Aurora-A (1:400; rabbit monoclonal; cat. no. 3079S; Cell Signaling Technology, Inc., Danvers, MA, USA), p-retinoblastoma (1:500; mouse monoclonal; cat. no. R6878-1ML) and $\beta$-actin (1:5,000; mouse monoclonal, cat. no. A2228-100UL; both from SigmaAldrich, St. Louis, MO, USA). Results were derived from three independent experiments with comparable outcomes.

Generation of tumorspheres. One thousand N1-hiPSCs 1GX (first generation xenografts) were cultured under non-adherent conditions using a defined stem cell medium (Stem Cell Technologies) for 10 days. The formation of tumorspheres was monitored and recorded using a Zeiss light microscope. After $48 \mathrm{~h}$ separate groups of tumorspheres were treated with the selective Aurora-A inhibitor, alisertib $(0.5 \mu \mathrm{M})$ for 8 days. Three independent experiments were performed with comparable outcomes.

Animal studies. Procedures established by the Institutional Animal Care and Use Committee (IACUC) of Mayo Clinic, based on the US NIH guidelines for the care and use of laboratory animals were adhered to for all experiments. Four-week-old SCID/beige mice were anesthetized by exposure to $3 \%$ isoflurane and $1 \times 10^{6} \mathrm{~N} 1$-hiPSCs were injected into their kidney capsule (three mice per each group). After 12 weeks, the mice were sacrificed and the tumor xenografts were processed for histology. To re-establish cell cultures from tumor explants, tumors tissues were excised from sacrificed animals, minced using sterile scissors, transferred to complete culture medium and fibroblast-free tumor cells were established by serial passages in culture. Animals were examined everyday and body weight and primary tumor size were assessed at least 1-2 times/week. Consistent distress and potential pain ( $>1$ day) were alleviated by euthanasia. If some of the animals were losing $>10 \%$ of their body weight, if blood was consistently observed in the urine or around the genitals of the mice, the mice were appropriately euthanized. When typical signs of distress including labored breathing and inactivity were consistently observed for $>1$ day, the animals were appropriately euthanized. When the primary tumor was $>2 \mathrm{~cm}$, the animals were sacrificed. Animals were euthanized using Pentobarbital (i.p., $100 \mathrm{mg} / \mathrm{kg}$ ) followed by cervical dislocation. The IACUC approved this study.

Experimental lung metastases. Four-week-old SCID/beige mice were anesthetized by exposure to $3 \%$ isoflurane and $1 \times 10^{6} \mathrm{~N} 1-$ hiPSCs $1 \mathrm{GX}$ were injected into their tail vein (three mice per each group) as previously described (14). After 4 weeks, the mice were sacrificed and lungs were isolated to detect metastatic lesions by employing a human-mitochondria specific antibody.

\section{Results and Discussion}

To determine the extent to which hiPSC reprogramming of somatic cells may induce the development of CIN, we 
A N1 human keratinocyte-derived IPS

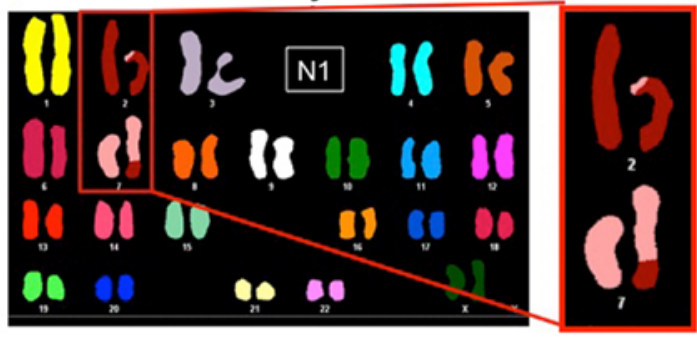

B DS1 human blood-derived IPS

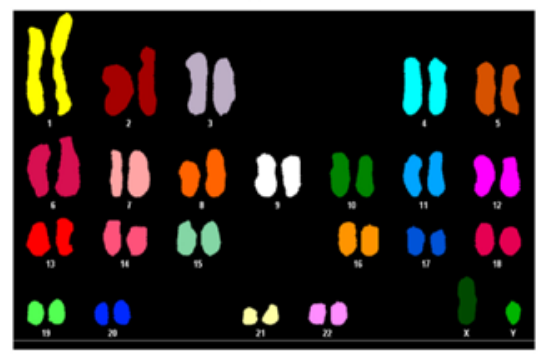

Figure 1. Genomic analysis of hiPSCs derived from keratinocytes and blood cells. (A) SKY technology performed in N1-HiPSCs exhibited a unique translocation between chromosomes 2 and 7. (B) SKY technology performed in DS1-hiPSCs exhibited a normal karyotype. hiPSCs, human induced pluripotent stem cells; SKY, spectral karyotyping.

generated hiPSCs from human keratinocytes and blood cells as previously described (16) and termed them N1-hiPSCs and DS1-hiPSCs, respectively. Chromosome analysis using spectral karyotyping (SKY) technology revealed that N1-hiPSCs were characterized by a translocation between chromosomes 2 and 7 (Fig. 1A), while DS1-hiPSCs exhibited a normal karyotype (Fig. 1B). This unique chromosome 2 and 7 translocation identified in N1-hiPSCs was uncommon since the predominant genetic changes found in hiPSCs involve structural and numerical changes in chromosomes 1, 12, 17 and $20(17,18)$. Due to the fact that chromosomal abnormalities are linked to mitotic defects during cell division, we analyzed the percentage of normal and aberrant mitoses in N1-hiPSCs. The counting of mitotic images revealed that $3.5 \%$ of total mitoses were characterized by multipolar mitotic spindles (Fig. 2A) that promote unequal chromosome segregation and CIN (19). Since hiPSC reprogramming is characterized by induction of genotoxic stress (20), we established whether development of multipolar mitoses was linked to genotoxic stress-induced centrosome amplification. N1-hiPSCs were treated with hydroxyurea (HU), a genotoxic agent that induces $\mathrm{G} 1 / \mathrm{S}$ cell cycle arrest and centrosome amplification in cancer cells lacking the p53-mediated G1/S cell cycle checkpoint (21). Following treatment with HU for $48 \mathrm{~h}, \mathrm{~N} 1-\mathrm{hiPSC}$ were arrested in the G1/S phase of the cell cycle (Fig. 2B). To determine whether G1/S cell cycle arrest was uncoupled from centrosome duplication, we analyzed the centrosome phenotype in N1-hiPSCs before and after HU treatment. The percentage of cells exhibiting centrosome amplification ( $>4$ centrioles) was increased in hiPSCs treated with HU (Fig. 2C and D), indicating that N1-hiPSCs exhibited a defective G1/S cell cycle checkpoint. Notably, after recovery from HU, N1-hiPSCs exhibited an exacerbation of centrosome amplification (Fig. 2C and D). One possible explanation is that
A N1 IPS cells

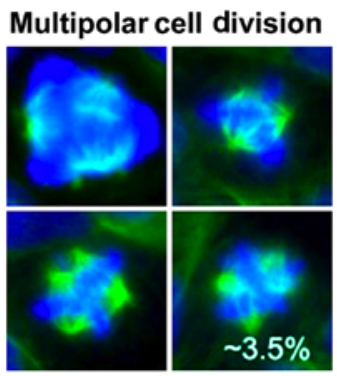

B

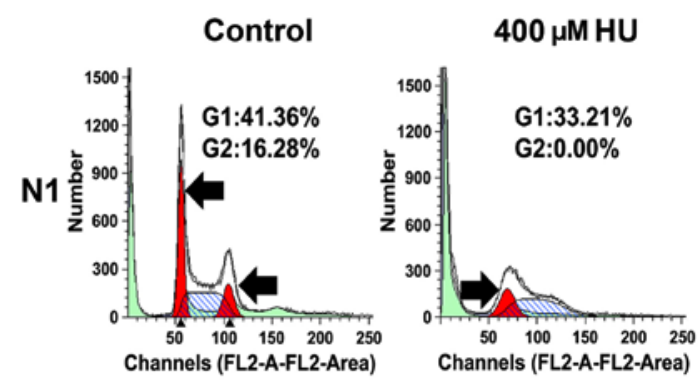

C N1 IPS Cells
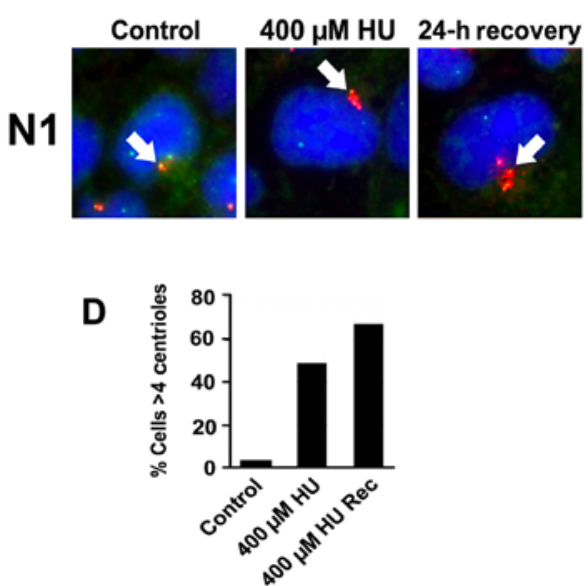

Figure 2. Analysis of G1/S cell cycle checkpoint integrity in N1-hiPSCs (A) Immunofluorescence analysis revealed multipolar mitotic spindles in N1-hiPSCs. Mitotic spindles were labeled in green with a monoclonal antibody targeting Aurora-A. Nuclei were labeled in blue with DAPI. (B) FACS analysis revealed that treatment with $400 \mu \mathrm{M}$ HU for $48 \mathrm{~h}$ induced the G1/S cell cycle arrest in N1-hiPSCs. (C) Immunofluorescence analysis revealed centrosome amplification in N1-hiPSCs treated with $400 \mu \mathrm{M} \mathrm{HU}$ for 48 and $24 \mathrm{~h}$ following $\mathrm{HU}$ washout. Centrioles were labeled in green with $20 \mathrm{H} 5$ centrin monoclonal antibody, pericentriolar material was labeled in red with a polyclonal antibody targeting pericentrin. Nuclei were labeled in blue with DAPI. (D) Graph displaying the average of the \% of N1-hiPSCs untreated (control), treated with $400 \mu \mathrm{M}$ HU for 48 and $24 \mathrm{~h}$ following $\mathrm{HU}$ washout from three independent experiments. hiPSCs, human induced pluripotent stem cells; HU, hydroxyurea.

after recovery from HU, N1-hiPSCs re-entered the cell cycle with amplified centrosomes leading to an increase of centrosome over-duplication. In view of the fact that development of centrosome amplification after genotoxic stress is functionally linked to abrogation of p53-mediated G1/S cell cycle checkpoint (21), we analyzed the integrity of p53 tumor-suppressor 


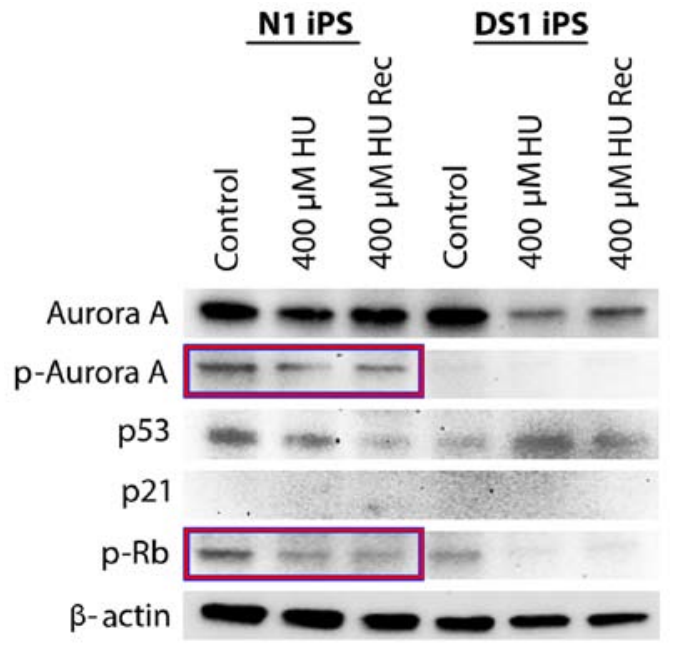

Figure 3. Expression of cell cycle checkpoint regulators in hiPSCs. Immunoblotting assay revealed the expression of p53, p21, p-Rb, total and phosphorylated Aurora-A before and following HU-induced genotoxic stress in N1-hiPSCs and DS1-hiPSCs. $\beta$-actin was used as a loading control. Results were derived from three independent experiments with comparable outcomes. hiPSCs, human induced pluripotent stem cells; HU, hydroxyurea.

signaling before and after HU treatment in N1-hiPSCs and DS1-hiPSCs (used as a control). N1-hiPSCs treated with HU exhibited low expression of p53 and did not exhibit a significant decrease of $\mathrm{Rb}$ phosphorylation (Fig. 3) that is critical to induce $\mathrm{Rb}$ activation, $\mathrm{G} 1 / \mathrm{S}$ cell cycle arrest and inhibition of centrosome amplification $(19,21)$. In contrast, DS1-hiPSCs treated with HU exhibited increased p53 expression and a significant decrease of $\mathrm{Rb}$ phosphorylation, indicating activation of the G1/S cell cycle checkpoint (Fig. 3). Due to neither N1-hiPSCs nor DS1-hiPSCs exhibiting increased expression of the p53 downstream target p21, we investigated the expression of the mitotic kinase Aurora-A that induces p53 degradation and centrosome amplification in cancer cells $(14,22)$. Notably, N1-hiPSCs exhibited high levels of total and phosphorylated (active kinase) Aurora-A before and after HU treatment, while Aurora-A levels were reduced in DS1-hiPSCs after HU treatment (Fig. 3), suggesting that aberrant expression/activation of Aurora-A is linked to abrogation of p53-mediated G1/S cell cycle checkpoint resulting in centrosome amplification, mitotic defects and CIN in hiPSCs. To establish the extent to which N1-hiPSCs overexpressing endogenous Aurora-A give rise to aggressive teratomas, N1-hiPSCs were injected into the kidney capsule of immunocompromised mice (Fig. 4A). After 12 weeks of injections, the animals were sacrificed and the tumors were isolated for histopathological analysis (Fig. 4B). N1-hiPSC-derived teratomas exhibited high tumor grade based on cells that exhibited non-uniform shapes and sizes and high nuclear pleomorphism. In addition, we identified in some tumor sections extensive regions of necrotic foci that are characteristic of proliferative malignant tumor cells (23). Notably, the majority of N1-hiPSC-derived teratoma cells exhibited duplicated and amplified centrosomes (Fig. 4C), demonstrating the linkage between an aggressive teratoma phenotype and dysregulation of the centrosome cycle responsible for CIN. Due to the fact that the tumor tissue analysis previously aforementioned revealed that N1-hiPSCderived tumors were more malignant than benign teratomas,

\section{A N1 IPS cells}

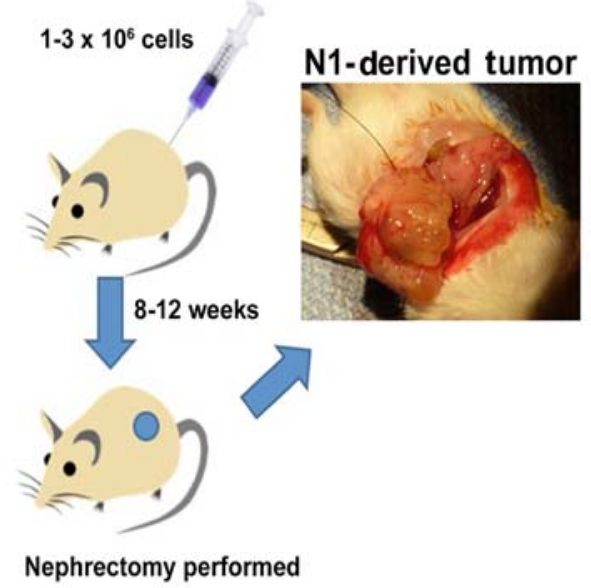

B Teratoma tumor tissue
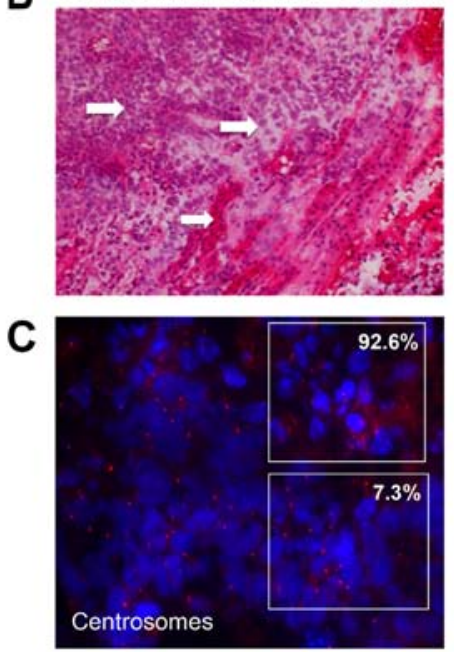

Figure 4. Development of teratomas from in vivo growth of N1-hiPSCs. (A) N1-hiPSCs $\left(1 \times 10^{6}\right)$ were injected into the kidney capsule of 4-week-old SCID/ beige mice to develop teratomas. (B) Animals were sacrificed after 12-week injections, and paraffin-embedded tumors were isolated for histopathological analysis. (C) Immunofluorescence analysis exhibiting centrosome phenotype in N1-derived teratomas. Centrosomes were labeled in red with a $\gamma$-tubulin monoclonal antibody. Nuclei were labeled in blue with DAPI. hiPSCs, human induced pluripotent stem cells.

we aimed to characterize their self-renewal and metastatic properties. N1-hiPSC-derived teratomas were excised and re-cultured cells were termed N1-hiPSCs 1GX (first generation xenografts). One thousand N1-HiPSCs 1GX were grown under non-adherent conditions for 10 days and successfully formed tumorspheres that represented an in vitro surrogate of self-renewal activity (Fig. 5A). To define the causal role of Aurora-A kinase activity in inducing N1-hiPSCs 1GX selfrenewal capacity, N1-hiPSCs 1GX tumorspheres were treated with the selective Aurora-A inhibitor alisertib. Treatment with alisertib significantly reduced the number and size of tumorspheres, demonstrating that Aurora-A kinase activity was required for the self-renewal capacity of teratoma cells (Fig. 5A). Subsequently, to determine the extent to which alisertib-mediated inhibition of self-renewal capacity was linked to impairment of $\mathrm{Rb}$ phosphorylation. Treatment of N1-hiPSCs 1GX tumorspheres with alisertib for $48 \mathrm{~h}$ reduced 
A

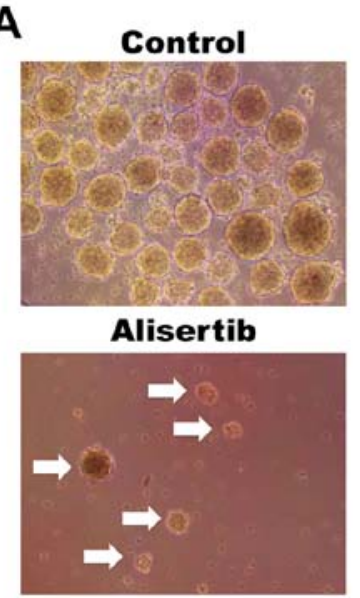

N1 1GX tumorspheres

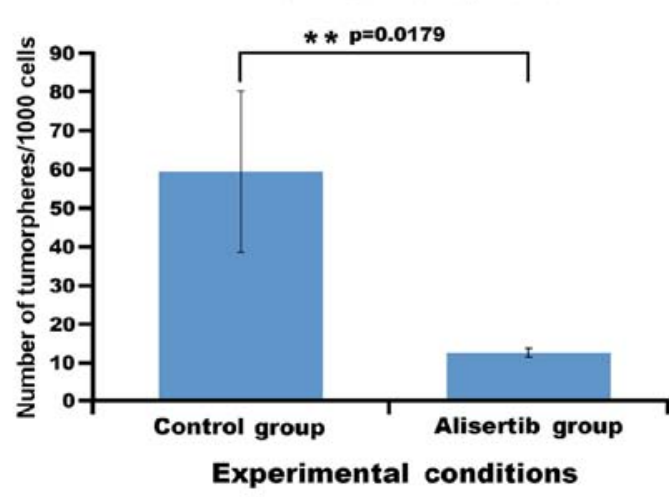

B

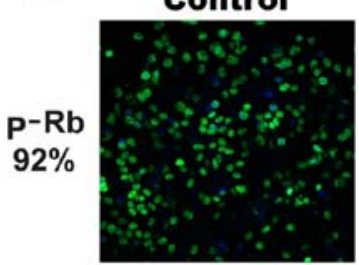

Alisertib
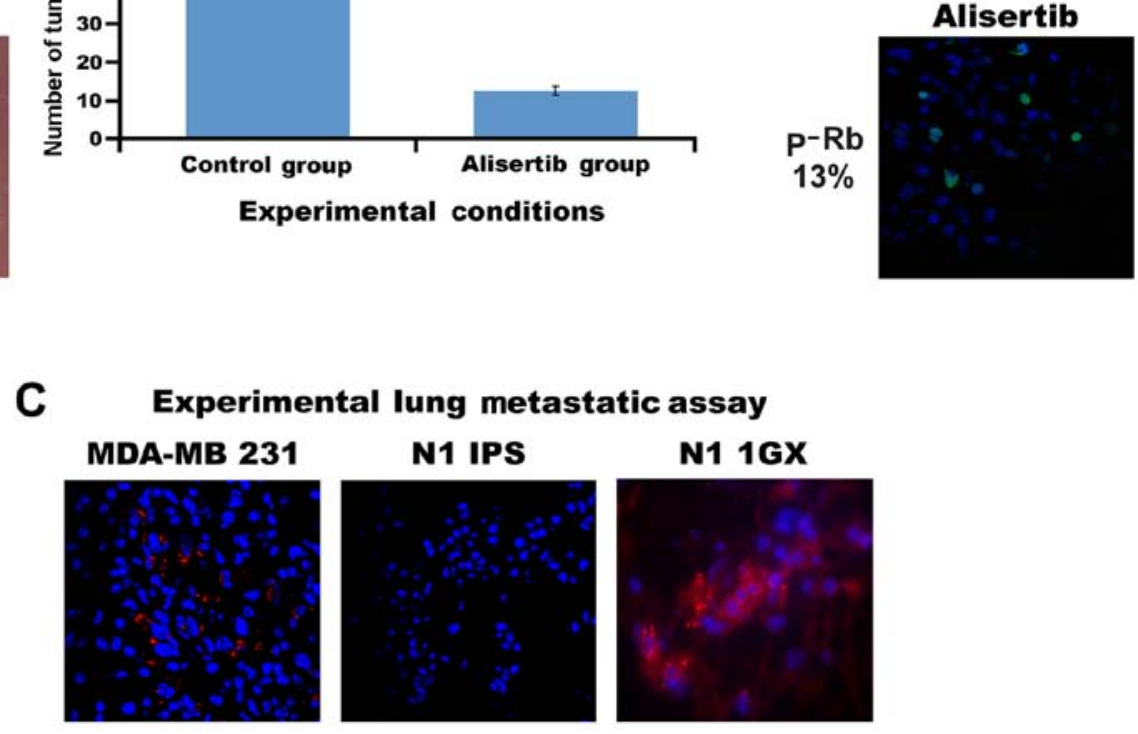

Figure 5. Tumor self-renewal and metastatic capacity of re-cultured teratoma cells. N1-hiPSC-derived teratomas were excised and re-cultured cells were termed N1-hiPSCs 1GX (first generation xenografts). (A) One thousand N1-hiPSCs 1GX were grown under non-adherent conditions and formed tumorspheres after 10 days in culture. After $48 \mathrm{~h}$ separate groups of tumorspheres were treated with the selective Aurora-A inhibitor, alisertib $(0.5 \mu \mathrm{M})$ for 8 days. The formation of tumorspheres was monitored and recorded using a Zeiss light microscope. Graph displaying the average of N1-hiPSCs 1GX tumorspheres before and after treatment with alisertib. The results represented the average from three independent experiments. (B) Immunofluorescence analysis displaying Rb phosphorylation in N1-hiPSCs 1GX tumorspheres before and after treatment with alisertib. Phosphorylated Rb was labeled in green with a p-Rb monoclonal antibody. Nuclei were labeled in blue with DAPI. The results represent the average from three independent experiments. (C) MDA-MB 231 cells (1x10 ${ }^{6}$, N1-hiPSCs and N1-hiPSCs 1GX were injected into the tail vein of 4-week-old SCID/beige mice and lung metastases were detected after 4 weeks of injections. Immunofluorescence analysis displaying lung sections where MDA-MB 231 and N1-hiPSCs 1GX are labeled in red using a human-mitochondria monoclonal antibody. Nuclei were labeled in blue with DAPI. Results were derived from three independent experiments with comparable outcomes. hiPSCs, human induced pluripotent stem cells; Rb, retinoblastoma.

nuclear Rb phosphorylation (Fig. 5B), indicating that Aurora-A kinase promoted N1-hiPSCs 1GX tumorsphere self-renewal capacity through phosphorylation and inactivation of the $\mathrm{Rb}$ tumor suppressor. Finally, to assess the malignant phenotype of N1-hiPSC-derived teratomas, N1-hiPSCs and N1-HiPSCs 1GX were injected into the tail vein of immunocompromised mice to develop experimental lung metastasis. Highly metastatic MDA-MB 231 breast cancer cells were used as a positive control (14). Only MDA-MB 231 cells and N1-HiPSCs 1GX developed experimental lung metastasis after 4 weeks of tail vein injections (Fig. 5C), demonstrating that hiPSCs with CIN give rise to aggressive teratomas in vivo. Collectively, these findings demonstrated a high risk for malignancy of human keratinocyte-derived hiPSCs that exhibited Aurora-A overexpression, centrosome amplification, loss of $\mathrm{Rb}$ function and CIN. Notably, rigorous quality-control tests, including comprehensive genomic integrity validation, analysis of $\mathrm{p} 53 /$ $\mathrm{Rb}$ tumor-suppressor pathways and Aurora-A kinase activity should be conducted before the clinical application of hiPSCs. Based on these findings, we propose that Aurora-A-targeted therapy could represent a promising prophylactic therapeutic strategy to decrease the likelihood of CIN and development of aggressive teratomas derived from hiPSCs.

\section{Acknowledgements}

The present study was supported by the USAMRMC BC022276, Intramural RECDA and The Nan Sayner Awards to A.B.D., NCI CA72836 to J.L.S., the Mayo Clinic Breast Cancer Specialized Program of Research Excellence (SPORE) NIH CA116201 to J.I., the Prospect Creek Foundation to E.G. and the Mayo Clinic Comprehensive Cancer Center. We also wish to acknowledge the Pathology Research Core facility of the Mayo Clinic School of Medicine and Science, for performing IHC assays and assisting us with the interpretation of the results.

\section{Competing interests}

The authors declare that they have no competing interests.

\section{References}

1. Takahashi K and Yamanaka S: Induction of pluripotent stem cells from mouse embryonic and adult fibroblast cultures by defined factors. Cell 126: 663-676, 2006.

2. Takahashi K, Tanabe K, Ohnuki M, Narita M, Ichisaka T, Tomoda $\mathrm{K}$ and Yamanaka S: Induction of pluripotent stem cells from adult human fibroblasts by defined factors. Cell 131: 861-872, 2007. 
3. Yu J, Vodyanik MA, Smuga-Otto K, Antosiewicz-Bourget J, Frane JL, Tian S, Nie J, Jonsdottir GA, Ruotti V, Stewart R, et al: Induced pluripotent stem cell lines derived from human somatic cells. Science 318: 1917-1920, 2007.

4. Pettinato G, Wen X and Zhang N: Formation of well-defined embryoid bodies from dissociated human induced pluripotent stem cells using microfabricated cell-repellent microwell arrays. Sci Rep 4: 7402, 2014.

5. Pettinato G, Vanden Berg-Foels WS, Zhang N and Wen X: ROCK inhibitor is not required for embryoid body formation from singularized human embryonic stem cells. PLoS One 9: e100742, 2014

6. Pettinato G, Wen $X$ and Zhang N: Engineering strategies for the formation of embryoid bodies from human pluripotent stem cells. Stem Cells Dev 24: 1595-1609, 2015.

7. Pettinato G, Ramanathan R, Fisher RA, Mangino MJ, Zhang N and Wen X: Scalable differentiation of human iPSCs in a multicellular spheroid-based 3D culture into hepatocyte-like cells through direct Wnt/ $\beta$-catenin pathway inhibition. Sci Rep 6 : 32888,2016

8. Ramanathan R, Pettinato G, Beeston JT, Lee DD, Wen X Mangino MJ and Fisher RA: Transplantation of human stem cell-derived hepatocytes in an animal model of acute liver failure. Surgery 158: 349-359, 2015.

9. Takahashi K and Yamanaka S: A decade of transcription factormediated reprogramming to pluripotency. Nat Rev Mol Cell Biol 17: 183-193, 2016

10. Riegler J, Ebert A, Qin X, Shen Q, Wang M, Ameen M, Kodo K, Ong SG, Lee WH, Lee G, et al: Comparison of magnetic resonance imaging and serum biomarkers for detection of human pluripotent stem cell-derived teratomas. Stem Cell Reports 6: 176-187, 2016.

11. Buganim Y, Markoulaki S, van Wietmarschen N, Hoke H, Wu T, Ganz K, Akhtar-Zaidi B, He Y, Abraham BJ, Porubsky D, et al: The developmental potential of iPSCs is greatly influenced by reprogramming factor selection. Cell Stem Cell 15: 295-309, 2014.

12. Holubcová Z, Matula P, Sedláčková M, Vinarský V, Doležalová D, Bárta T, Dvořák P and Hampl A: Human embryonic stem cells suffer from centrosomal amplification. Stem Cells 29: 46-56, 2011.

13. Marión RM, Strati K, Li H, Murga M, Blanco R, Ortega S, Fernandez-Capetillo O, Serrano $M$ and Blasco MA: A p53-mediated DNA damage response limits reprogramming to ensure iPS cell genomic integrity. Nature 460: 1149-1153, 2009.
14. D'Assoro AB, Liu T, Quatraro C, Amato A, Opyrchal M, Leontovich A, Ikeda Y, Ohmine S, Lingle W, Suman V, et al: The mitotic kinase Aurora: A promotes distant metastases by inducing epithelial-to-mesenchymal transition in $\mathrm{ER} \alpha^{+}$breast cancer cells. Oncogene 33: 599-610, 2014.

15. Lee DF, Su J, Ang YS, Carvajal-Vergara X, Mulero-Navarro S, Pereira CF, Gingold J, Wang HL, Zhao R, Sevilla A, et al: Regulation of embryonic and induced pluripotency by aurora kinase-p53 signaling. Cell Stem Cell 11: 179-194, 2012.

16. Ohmine S, Squillace KA, Hartjes KA, Deeds MC, Armstrong AS Thatava T, Sakuma T, Terzic A, Kudva Y,Ikeda Y: Reprogrammed keratinocytes from elderly type 2 diabetes patients suppress senescence genes to acquire induced pluripotency. Aging (Albany NY) 4: 60-73, 2012.

17. Draper JS, Moore HD, Ruban LN, Gokhale PJ and Andrews PW: Culture and characterization of human embryonic stem cells Stem Cells Dev 13: 325-336, 2004.

18. Maitra A, Arking DE, Shivapurkar N, Ikeda M, Stastny V, Kassauei K, Sui G, Cutler DJ, Liu Y, Brimble SN, et al: Genomic alterations in cultured human embryonic stem cells. Nat Genet 37: 1099-1103, 2005.

19. D'Assoro AB, Busby R, Acu ID, Quatraro C, Reinholz MM, Farrugia DJ, Schroeder MA, Allen C, Stivala F, Galanis E and Salisbury JL: Impaired p53 function leads to centrosome amplification, acquired ERalpha phenotypic heterogeneity and distant metastases in breast cancer MCF-7 xenografts. Oncogene 27: 3901-3911, 2008.

20. Ruiz S, Lopez-Contreras AJ, Gabut M, Marion RM, GutierrezMartinez P, Bua S, Ramirez O, Olalde I, Rodrigo-Perez S, Li H, et al: Limiting replication stress during somatic cell reprogramming reduces genomic instability in induced pluripotent stem cells. Nat Commun 6: 8036, 2015.

21. D'Assoro AB, Busby R, Suino K, Delva E, AlmodovarMercado GJ, Johnson H, Folk C, Farrugia DJ, Vasile V, Stivala F, et al: Genotoxic stress leads to centrosome amplification in breast cancer cell lines that have an inactive G1/S cell cycle checkpoint. Oncogene 23: 4068-4075, 2004.

22. Katayama H, Sasai K, Kawai H, Yuan ZM, Bondaruk J, Suzuki F, Fujii S, Arlinghaus RB, Czerniak BA and Sen S: Phosphorylation by aurora kinase A induces Mdm2-mediated destabilization and inhibition of p53. Nat Genet 36: 55-62, 2004.

23. Zhou J, Schmid T, Schnitzer S and Brüne B: Tumor hypoxia and cancer progression. Cancer Lett 237: 10-21, 2006. 